九州大学学術情報リポジトリ

Kyushu University Institutional Repository

\title{
Multi-species Generation Strategy-Based Vegetation Evolution
}

YU, Jun

Institute of Science and Technology, Niigata University

高木，英行

Faculty of Design, Kyushu University

http://hdl. handle. net/2324/4488108

出版情報: Congress on Evolutionary Computation, pp.3475-3480，2020-07-19. IEEE バージョン：

権利関係 : $\odot 2006$ IEEE. Personal use of this material is permitted. Permission from IEEE must be obtained for all other uses, in any current or future media, including reprinting/republishing this material for advertising or promotional purposes, creating new collective works, for resale or redistribution to servers or lists, or reuse of any copyrighted component of this work in other works. 


\section{Multi-species Generation Strategy-Based Vegetation Evolution}

\author{
Jun YU \\ Institute of Science and Technology \\ Niigata University \\ Niigata, Japan \\ Email: yujun@ ie.niigata-u.ac (dot)jp
}

\author{
Hideyuki TAKAGI \\ Faculty of Design \\ Kyushu University \\ Fukuoka, Japan \\ Email: takagi @ design.kyushu-u.ac (dot)jp
}

\begin{abstract}
We propose a multi-species generation strategy to increase the diversity of seed individuals produced in the maturity operation of vegetation evolution (VEGE). Since the breeding patterns of real plants can be roughly divided into sexual reproduction and asexual one, the proposed strategy additionally introduces two different methods to simulate these two patterns. As our preliminary attempt of the simulation, a mature individual is splattered randomly in the neighbor local area of its parent individual with Gaussian distribution probability to simulate asexual reproduction, while a mature individual is generated by crossing randomly selected two different parent individuals to simulate sexual reproduction. Our proposed strategy consists of these two new reproduction methods and that of our original VEGE, and each mature individual in every generation randomly selects one of these three methods to generate seed individuals, which is analogous to different plant species using different mechanisms to breed. To evaluate the performance of our proposed strategy, we compare VEGE and (VEGE + the proposed generation strategy) on 28 benchmark functions of three different dimensions from the CEC 2013 test suit with 30 independent trial runs. The experimental results have confirmed that the proposed strategy can increase the diversity of seed individuals, accelerate the convergence of VEGE significantly, and become effective according to the increase of dimensions.

Index Terms-Evolutionary Computation, Vegetation Evolution, Multi-species Generation Strategy, Optimization
\end{abstract}

\section{INTRODUCTION}

Since the genetic algorithm (GA) [1] triggered a new wave of optimization technology research, population-based evolutionary computation (EC) algorithms have gradually attracted more and more widespread attention and successfully solved many complicated real-world problems. So far, many dozens of powerful EC algorithms borrowing different ideas from biological group behavior or natural phenomena have been proposed and achieved satisfactory results, such as particle swarm optimization (PSO) [2], differential evolution (DE) [3], and others [4]-[7]. Most researchers focus on introducing various effective strategies to further improve the performance of these EC algorithms [8]-[10]. A small number of researchers also try to approximate the fitness landscape of optimization problems and use it to reduce evaluation costs and accelerate EC search [11]-[13]. Thus, finding ways of

This work was supported in part by Grant-in-Aid for Scientific Research (18K11470) accelerating convergence with lower cost consumption has become one of the hottest topics.

Vegetation evolution (VEGE) is a new member of the EC family and shows a stronger optimization ability compared with standard PSO and DE [14]. The core innovation of VEGE is to simulate two different growth stages of plants, which we call the growth stage and the maturity stage, to find the global optimum. Its biggest feature is that each of individuals has these two cyclical stages, thus exploration and exploitation are alternately emphasized to maintain their balance well. We designed a series of controlled experiments, investigated the impact of each VEGE operation on performance, and found that some operations did not achieve the expected results [15]. This finding motivated us to conduct the research described in this paper, wherein we propose a new strategy to overcome the defects found.

The main objective of this paper is to propose a multispecies generation strategy to increase the diversity of seed individuals in the maturity operation. Inspired by the breeding mechanisms of real plants, two additional methods are introduced to simulate sexual reproduction and asexual reproduction, respectively. The proposed strategy thus provides total three different methods for generating seed individuals, including the original generation method of VEGE. Each mature individual selects one of the three methods randomly to generate seed individuals, which is as if different plant species were using different mechanisms to breed. The secondary objective is to analyze the effectiveness and applicability of the proposed strategy and point out some potential topics to discuss.

\section{Vegetation Evolution}

Although there are many kinds of plants in nature and each has its own unique mechanism to ensure the survival of its species, we have observed that their life cycle is similar; that is: the plants grow from seeds, mature, generate their new seeds, and disperse them. Some seeds germinate in suitable environment and begin a new round of their life cycle; others which are not suited to the environment are eliminated and cannot grow.

VEGE simulates these two growth stages, i.e. the growth period and the maturity period, repeatedly to improve the 
accuracy of candidate solutions and eventually converge to the global optimum. Corresponding to the optimization framework of VEGE, the growth period can be considered as a local search period for exploitation, while the maturity period can be considered as a global search period for exploration. Fig. 1 illustrates an example of the abstract life cycle of real plants, which can help to grasp the core idea of VEGE optimization visually.

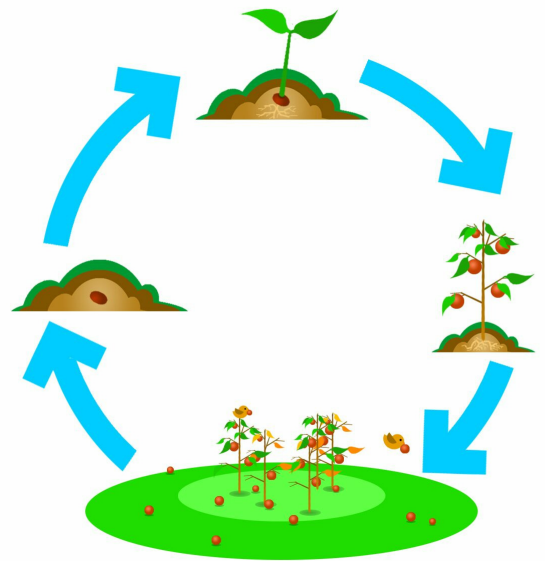

Fig. 1. The abstract lifecycle of real plants. We divide it into two different stages subjectively, the growth period and the maturity period.

Like most EC algorithms, VEGE randomly generates multiple individuals to form an initial population, all individuals search their local areas independently, and they generate their offspring individuals in their neighborhood along a randomly selected direction, which can be seen as an analog to plant growth. Offspring individuals and their parent ones are compared in the growth period, and only better offspring individuals can replace their parent individuals. When this growth operation is repeatedly applied until the predetermined maximum number of growths, each individual becomes mature, and enters the maturity period.

The maturity period simulates plant breeding. Since the parent-offspring relationship in the maturity period is oneto-many, each individual generates multiple seed individuals based on the Eq.(1) in the Table I. All individuals, i.e. both parent individuals and seed individuals, are ranked according to their fitness, and the top $P S$ ( $P S$ : population size) individuals survive to the next generation.

Finally, the survived individuals begin their new life cycle until a termination condition is satisfied. Fig. 2 shows the general VEGE optimization process that mainly consists of initialization, the growth operation, the mature operation, and selection. More detailed information of the VEGE algorithm is in the reference [14].

\section{Multi-Species Generation Strategy}

Many animals throughout history have perished when their environments changed, where plants have persisted on the earth owing to their mechanisms for adapting to harsh environments. VEGE roughly approximates and simulates the life

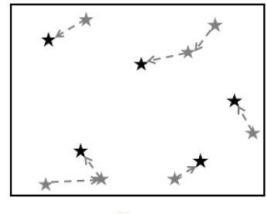

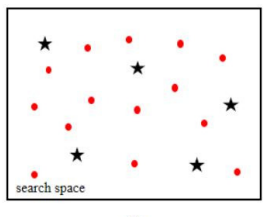

(b)

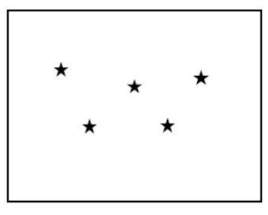

(c)
Fig. 2. The search process of the VEGE algorithm. (a) Initial population is randomly generated; dotted arrows indicate individuals growing toward higher potential in local areas when the generated offspring individuals are better than their parents. (b) Multiple seed individuals (red dots) are generated by each individual. (c) Individuals in the new generation are selected from all individuals in the step (b). Steps (b) and (c) are iterated until a termination condition is satisfied.

cycle of real plants to demonstrate powerful optimization performance. However, plants have actually evolved many more complex mechanisms to adapt to their various environments; they can provide us with new inspirations to further improve the performance of VEGE by integrating into it more observed mechanisms from real plants.

Based on the results of our previous analysis [15], we found that the maturity operation plays an important role and greatly affects the performance. Unfortunately, the original VEGE only uses the Eq. (1) in the Table I to generate seed individuals and disperse them. Consequently, it can easily fall into local minima which are difficult to escape for some types of problems because it is difficult for the original VEGE using a single generation method (Eq. (1)) to provide sufficient diversity.

We noticed that real plants have many ways to generate their seeds, and the more primitive the plant is, the more the ratio of asexual reproduction becomes. For example, algae and fungi generate offspring with asexual reproduction, while plants with higher degrees of evolution, such as wheat, corn, and cucumber, generate offspring using sexual reproduction. There are some higher level plants with strong adaptability in which the two breeding methods are alternated. Lotus roots and reeds are the most representative. Although there are various breeding strategies, we can roughly classify them as either sexual reproduction or asexual one. The proposed strategy is thus expected to generate more diverse seed individuals in the maturity operation, allowing it to overcome the abovementioned drawbacks, by additionally simulating these two breeding methods.

Since the probability density function curve of a Gaussian distribution is bell-shaped, we can set a mature individual at the center of a Gaussian distribution and adjust the variance to tune the area size for exploration. This procedure can be used to simulate asexual reproduction of plants; that is, the mature individual generates many seed individuals which are dispersed into the surrounding areas, with greater dispersal distances happening with less frequency. The Eq. (2) in the Table I illustrates how seed individuals can thus be generated by asexual reproduction.

The key to simulating sexual reproduction lies in the exchange of genetic information between individuals. Although 
GA already provided multiple ways for genetic exchange, such as single-point crossover, multi-point crossover, and binomial crossover, we finally decided to use the binomial crossover to simulate the sexual reproduction of plants because it can maximize the genetic difference between offspring individuals and their parent individual. Thus, the Eq. (3) in the Table I can be used to generate seed individuals by means of sexual reproduction. Clearly, other methods for simulate sexual reproduction could also be used.

Table I shows three methods for generating seed offspring in our proposed strategy. When individuals in each generation become mature, they randomly select a method among three methods provided to generate their seed individuals, which can be compared to different plant species using different ways to breed. In brief, the proposal focuses only on modifications to the maturity operation without involving any other operations. Algorithm 1 summarizes the optimization process of integrating the proposed strategy into the standard VEGE.

TABLE I

PROPOSED STRATEGY CONSISTS OF THREE METHODS FOR GENERATING SEED INDIVIDUALS

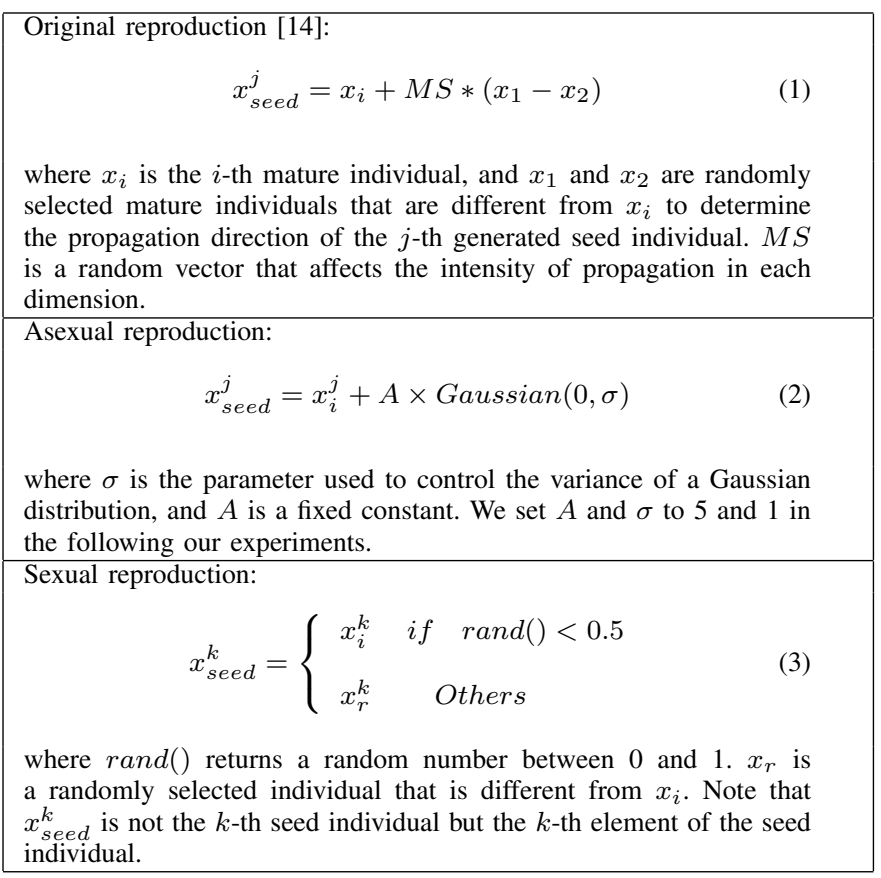

\section{ExPERimental EVAluations}

We design a set of control experiments to analyze the performance of our proposed multi-species generation strategy, and select 28 benchmark functions from the CEC 2013 test suite [16] that are specifically used for single-objective performance evaluation and include a variety of features, such as, shifted, rotated, unimodal and multi-modal. We run VEGE and (VEGE + our proposed generation strategy) on these functions with three different dimensions, i.e. 2-D, 10-D, and 30-D, and each function run independently 30 times to avoid contingency and reduce errors. The parameter settings of the standard VEGE

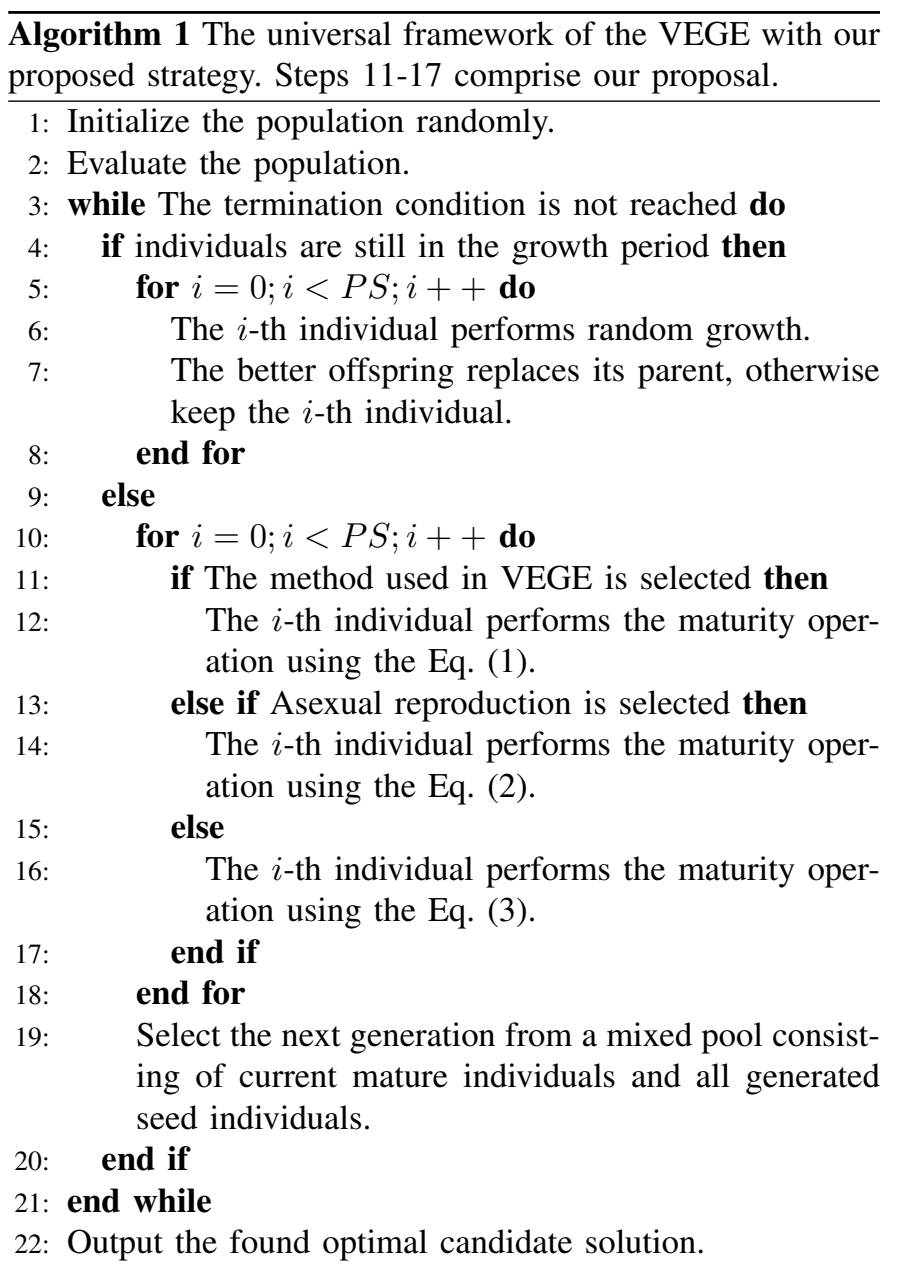

used in our experiments are described in the Table II, and their detailed meaning of these parameters are in the paper [14].

TABLE II

THE PARAMETER SETTINGS OF USED VEGE.

\begin{tabular}{|l|c|}
\hline population size & 100 \\
\hline for 2-D, 10-D, and 30-D search & 100 \\
\hline number of growth operations & 5 \\
\hline growth radius & 500 \\
\hline $\begin{array}{l}\text { total seed individuals } \\
\text { for 2-D, 10-D, and 30-D search }\end{array}$ & a random number in [-1,1] \\
\hline moving scaling $M S$ & a random number in $[-2,2]$ \\
\hline stop condition; max. \# of fitness evaluations & $2000 \times \mathrm{D}$ \\
\hline
\end{tabular}

We use the maximum number of fitness evaluations to terminate the experiment for a fair comparison, and apply the Wilcoxon signed-rank test to check the significance of the difference between VEGE and (VEGE + our proposed generation strategy) at the termination condition. The results of the statistical tests are shown in the Table III, and the Fig. 3 shows the average convergence curve of 28 functions in 30-D.

\section{Discussions}

We first begin our discussion by reviewing the benefits offered by the proposed multi-species generation strategy. The maturity operation is designed to provide diverse seed 
TABLE III

STATISTICAL TEST RESULTS OF THE WILCOXON SIGNED-RANK TEST FOR THE AVERAGE FOUND OPTIMUM OF 30 TRIAL RUNS AT THE STOP CONDITION. $A \gg B$ AND $A>B$ MEAN THAT $A$ IS SIGNIFICANTLY BETTER THAN $B$ WITH SIGNIFICANT LEVELS OF $1 \%$ AND 5\%, RESPECTIVELY. $A \approx B$ MEANS THAT THERE IS NO SIGNIFICANT DIFFERENCE BETWEEN THEM ALTHOUGH $A$ IS BETTER THAN $B$. PROPOSAL: (VEGE + OUR PROPOSED GENERATION STRATEGY).

\begin{tabular}{|c|c|c|c|}
\hline & $2 \mathrm{D}$ & 10D & 30D \\
\hline$F 1$ & Proposal $\gg$ VEGE & Proposal $\gg$ VEGE & Proposal $\gg$ VEGE \\
\hline$F 2$ & Proposal $\approx$ VEGE & Proposal $\gg$ VEGE & Proposal $\gg$ VEGE \\
\hline$F 3$ & Proposal $\approx$ VEGE & Proposal $\gg$ VEGE & Proposal $\gg$ VEGE \\
\hline$F 4$ & Proposal $\approx$ VEGE & VEGE $\approx$ Proposal & Proposal $\gg$ VEGE \\
\hline$F 5$ & Proposal > VEGE & Proposal $\gg$ VEGE & Proposal $\gg$ VEGE \\
\hline$F 6$ & VEGE $>$ Proposal & Proposal $\gg$ VEGE & Proposal $\gg$ VEGE \\
\hline$F 7$ & Proposal $\approx$ VEGE & Proposal 》 VEGE & Proposal $\gg$ VEGE \\
\hline$F 8$ & Proposal $\approx$ VEGE & VEGE $\approx$ Proposal & VEGE $\approx$ Proposal \\
\hline$F 9$ & Proposal $\approx$ VEGE & Proposal $\gg$ VEGE & Proposal $\gg$ VEGE \\
\hline$F_{10}$ & Proposal $\approx$ VEGE & Proposal $\gg$ VEGE & Proposal $\gg$ VEGE \\
\hline$F_{11}$ & Proposal $\gg$ VEGE & Proposal $\gg$ VEGE & Proposal 》 VEGE \\
\hline$F_{12}$ & Proposal $\approx$ VEGE & Proposal $\gg$ VEGE & Proposal 》 VEGE \\
\hline$F_{13}$ & VEGE $\approx$ Proposal & Proposal $\gg$ VEGE & Proposal $\gg$ VEGE \\
\hline$F_{14}$ & Proposal $\gg$ VEGE & Proposal $\gg$ VEGE & Proposal $\gg$ VEGE \\
\hline$F_{15}$ & VEGE $\approx$ Proposal & Proposal $\gg$ VEGE & Proposal $\gg$ VEGE \\
\hline$F_{16}$ & VEGE $\approx$ Proposal & VEGE $\approx$ Proposal & Proposal $\approx$ VEGE \\
\hline$F_{17}$ & Proposal $\approx$ VEGE & Proposal $\gg$ VEGE & Proposal $\gg$ VEGE \\
\hline$F_{18}$ & VEGE $\approx$ Proposal & Proposal $\gg$ VEGE & Proposal $\gg$ VEGE \\
\hline$F_{19}$ & Proposal $\approx$ VEGE & Proposal $\gg$ VEGE & Proposal $\gg$ VEGE \\
\hline$F_{20}$ & VEGE $\approx$ Proposal & Proposal $\gg$ VEGE & Proposal 》 VEGE \\
\hline$F_{21}$ & Proposal $\gg$ VEGE & Proposal $\gg$ VEGE & Proposal 》 VEGE \\
\hline$F_{22}$ & Proposal $\gg$ VEGE & Proposal $\gg$ VEGE & Proposal $\gg$ \\
\hline$F_{23}$ & Proposal $\approx$ VEGE & Proposal $\gg$ VEGE & Proposal $\gg$ VEGE \\
\hline$F_{24}$ & VEGE $\approx$ Proposal & Proposal $\gg$ VEGE & Proposal $\gg$ VEGE \\
\hline$F_{25}$ & VEGE $\approx$ Proposal & Proposal $\gg$ VEGE & Proposal 》 VEGE \\
\hline$F_{26}$ & Proposal > VEGE & VEGE $\approx$ Proposal & Proposal $\gg$ VEGE \\
\hline$F_{27}$ & Proposal $\approx$ VEGE & Proposal $\gg$ VEGE & Proposal $\gg$ VEGE \\
\hline$F_{28}$ & Proposal > VEGE & Proposal $\gg$ VEGE & Proposal $\gg$ VEGE \\
\hline
\end{tabular}

individuals and to disperse them as far as possible into different areas. The standard VEGE uses differential information to generate seed individuals and determine their propagation direction (Eq.(1)). Although this method uses the distribution information of individuals indirectly from the distance between randomly chosen two differential individuals and adaptively determine the distance to the generated offspring individual, individuals become more and more similar according to the convergence of the population, and it becomes difficult to determine effective and diversified directions for propagation. This situation makes individuals difficult to escape when they gradually converge to a local minimum. How to always provide diverse seed individuals with the maturity operation has thus become a key research topic, especially in the later stages of convergence.

Through observing the breeding patterns of real plants, we realized that these patterns could be introduced into VEGE to increase the diversity of seed individuals. As a preliminary attempt, we roughly divide all breeding patterns into two main categories: asexual reproduction and sexual reproduction, and the proposed strategy uses Gaussian distribution and binomial crossover to simulate these two new breeding patterns. Thus, the proposal can provide many different ways to generate seed individuals to avoid to be trapped in local areas, and individ- uals can freely switch among different offspring generation methods in each search generation to increase randomness and diversity by playing the role of different plant species. Furthermore, the proposal does not need any additional fitness calculations, and the increased CPU time required for randomly selecting the offspring generation method is also negligible. Finally, we only use the proposed offspring generation strategy to replace the original generation strategy in the maturity operation without touching other operations, so our proposed strategy is low cost and easy to use.

Secondly, we discuss the scalability of the proposed strategy. We used Gaussian distribution and binomial crossover in this paper, but any other methods for simulating sexual reproduction and asexual one can be integrated into our proposed strategy. For example, we can simulate asexual reproduction by dispersing seed individuals into neighbor areas with equal probability and sexual reproduction by using the exponential crossover. Since real plants have a great variety of breeding patterns, we may obtain some new inspirations from them and can further improve the VEGE performance. The success of the modification in this paper implies that VEGE still has a lot of room for further improvement. Real plants have many different ways to disperse their seeds, e.g. wind dispersal, animal carrying, and others. We can also introduce these mechanisms to simulate the plant life cycle more accurately. Thus, how to disperse seed individuals efficiently is also a subject worthy of study.

Next, we give some open topics for discussions. The proposed strategy selects one of multiple generation methods and generates seed individuals with equal probability. This approach can increase the randomness but may also reduce the search efficiency and convergence speed, especially for complex noisy problems. Thus, how to select a reasonable offspring generation method instead of a random selection used in this paper is also a promising topic. For example, it is one possible approach to record the performance improvement of each offspring generation method and give higher selection probability to more effective method. Selecting an offspring generation method based on the information collected during the optimization process, e.g. individual distribution density and proportion of evolved individuals, is other possible approach. In addition, as improvement is not limited to the maturity operation, we can borrow more ideas from real plants to improve other operations, such as the growth operation and the selection operation.

Finally, we applied the Wilcoxon signed-rank test to check the significant differences between the original VEGE and (the original VEGE + our proposed strategy). The statistical test results and average convergence curves of the Fig. 3 confirm that diverse seed individuals generated by our proposed strategy can accelerate the convergence speed and avoid falling into local areas. This acceleration becomes more effective according to the increase of dimensions. It may be the reason why the proposal can disperse seed individuals more widely in the higher dimensional search space and therefor quickly find potential areas. Since each mature individual 
has the opportunity to choose a different offspring generation method in every generation, it also makes the population not easily fall into local areas. However, the proposal has no effect on $F_{8}$ and $F_{16}$ regardless their dimensions, and their average convergence curves in the Fig. 3 are similar, but the performance fluctuations are relatively high. This may be due to their so many local minima that it is also difficult for the proposed strategy to provide sufficient diversity of seed individuals, or even reduce the convergence speed. We will continue to analyze the underlying reasons in order to further improve the performance of VEGE in the near future.

\section{CONCLUSION}

Inspired by breeding patterns of real plants, we propose a multi-species generation strategy to increase the diversity of seed individuals in the maturity period by providing multiple different offspring generation methods. We confirmed that our proposed strategy can improve the convergence speed effectively, and the effect is more obvious for higher dimensional tasks.

In our future work, we will continue to observe the breeding patterns of real plants and introduce them into VEGE, and develop an adaptive switching strategy to select an appropriate generation method based on optimization progress and the characteristics of optimization problems.

\section{REFERENCES}

[1] J. H. Holland, "Outline for a logical theory of adaptive systems," $J$. Assoc. Comput. Mach., vol. 3, pp. 297-314, 1962.

[2] J. Kennedy and R. Eberhart, "Particle swarm optimization," in IEEE International Conference on Neural Networks, 1995, pp. 1942-1948.

[3] R. Storn and K. Price, "Differential evolution - a simple and efficient heuristic for global optimization over continuous spaces," Journal of Global Optimization, vol. 11, no. 4, pp. 341-359, 1997.

[4] K. Dervis and B. Bahriye, "A powerful and efficient algorithm for numerical function optimization: Artificial bee colony (abc) algorithm," Journal of Global Optimization, vol. 39, no. 3, pp. 459-471, 2007.

[5] X. Yang and S. Deb, "Cuckoo search via lvy flights," in 2009 World Congress on Nature and Biologically Inspired Computing, NABIC 2009 - Proceedings, 2009, pp. 210-214.

[6] Y. Tan and Y. Zhu, "Fireworks algorithm for optimization," in The First International Conference on Swarm Intelligence, 2010, pp. 355-364.

[7] G. Amir, Hossein and A. Amir, Hossein, "Krill herd: A new bioinspired optimization algorithm," Communications in Nonlinear Science and Numerical Simulation, vol. 17, no. 12, pp. 4831-4845, 2012.

[8] J. Yu, Y. Pei, and H. Takagi, "Competitive strategies for differential evolution," in The 2018 IEEE International Conference on Systems, Man, and Cybernetics, 2018, pp. 268-273.

[9] J. Yu and H. Takagi, "Acceleration for fireworks algorithm based on amplitude reduction strategy and local optima-based selection strategy," in The 8th International Conference on Swarm Intelligence, 2017, pp. $477-484$.

[10] J. Yu, Y. Tan, and H. Takagi, "Scouting strategy for biasing fireworks algorithm search to promising directions," in The Genetic and Evolutionary Computation Conference Companion, 2018, pp. 99-100.

[11] J. Yu, Y. Pei, and H. Takagi, "Accelerating evolutionary computation using estimated convergence points," in 2016 IEEE Congress on Evolutionary Computation, 2016, pp. 1438-1444.

[12] Y. Jin, M. Olhofer, and B. Sendhoff, "A framework for evolutionary optimization with approximate fitness functions," IEEE Transactions on Evolutionary Computation, vol. 6, no. 5, pp. 481-494, 2002.

[13] Y. Jin, "A comprehensive survey of fitness approximation in evolutionary computation," Soft Computing, vol. 9, no. 1, pp. 3-12, 2005.

[14] J. Yu, "Study on acceleration for evolutionary computation," Ph.D. dissertation, Kyushu University, Fukuoka, Japan, 2019. [Online]. Available: https://catalog.lib.kyushu-u.ac.jp/opac_download_ $\mathrm{md} / 2534456 /$ design0262.pdf

[15] J. Yu and H. Takagi, "Performance analysis of vegetation evolution," in The 2019 IEEE International Conference on Systems, Man, and Cybernetics, 2019, pp. 2214-2219.

[16] J. Liang, B. Qu, P. Suganthan, and A. G. Hernández-Díaz, "Problem definitions and evaluation criteria for the cec 2013 special session on real-parameter optimization," 2013. [Online]. Available: http://al-roomi. org/multimedia/CEC_Database/CEC2013/RealParameterOptimization/ CEC2013_RealParameterOptimization_TechnicalReport.pdf

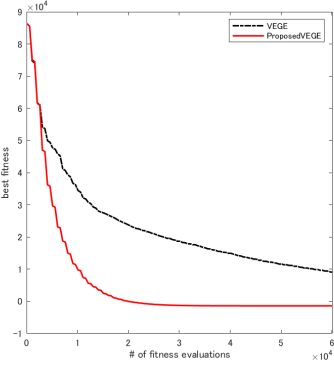

$F_{1}$

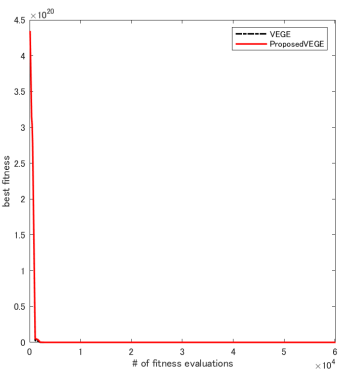

$F_{3}$

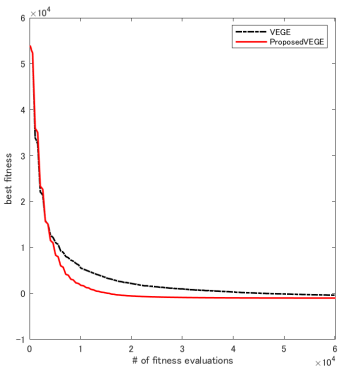

$F_{5}$

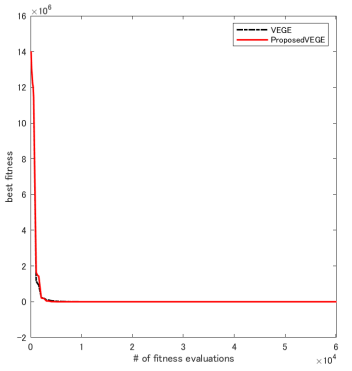

$F_{7}$

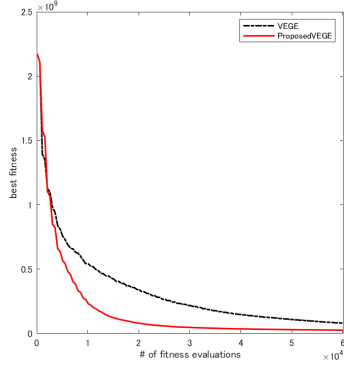

$F_{2}$

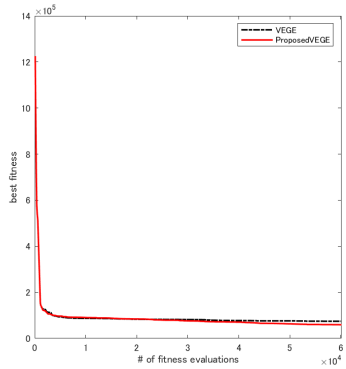

$F_{4}$

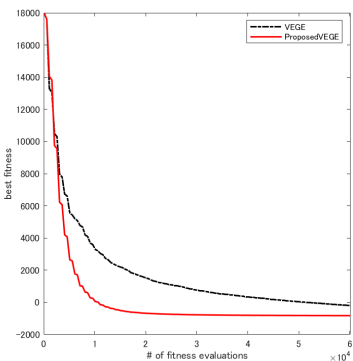

$F_{6}$

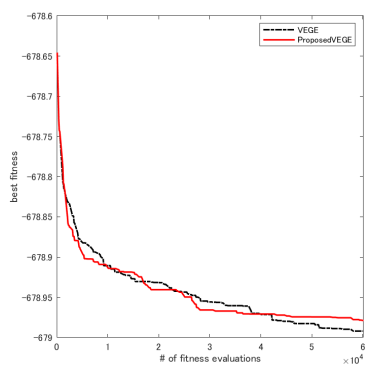

$F_{8}$ 


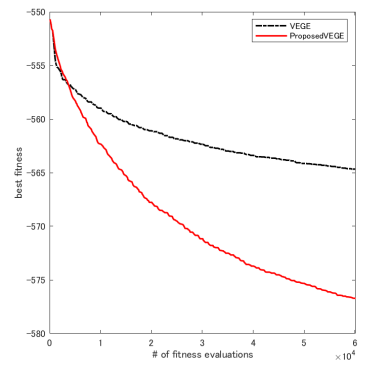

$F_{9}$

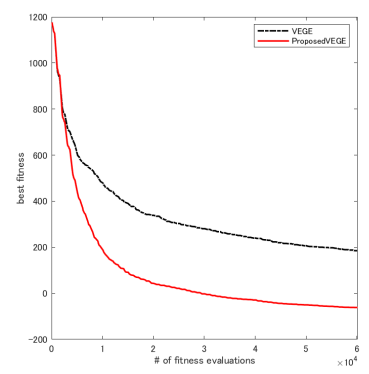

$F_{13}$

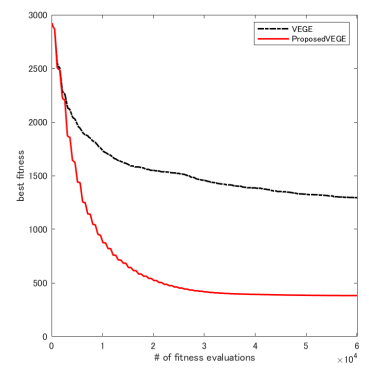

$F_{17}$

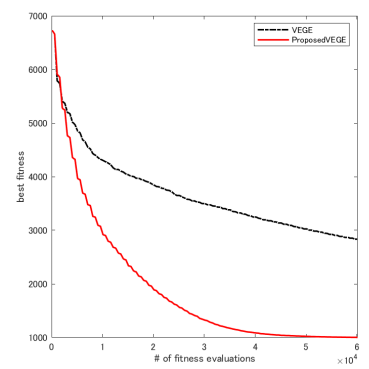

$F_{21}$

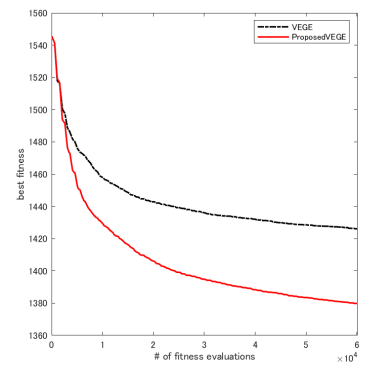

$F_{25}$

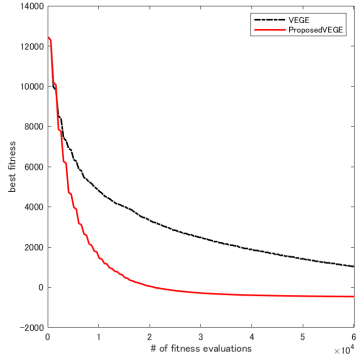

$F_{10}$

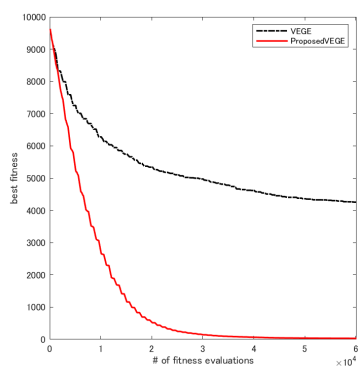

$F_{14}$

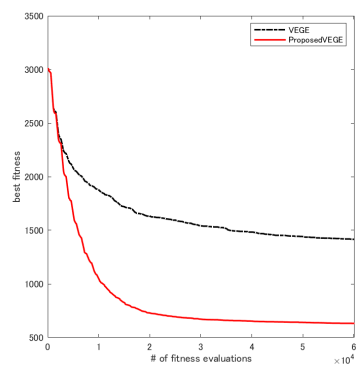

$F_{18}$

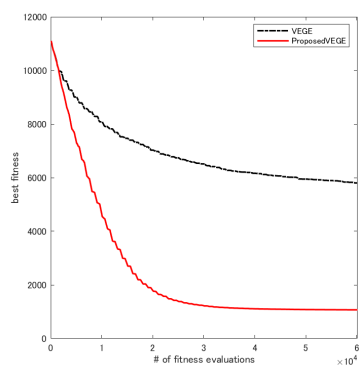

$F_{22}$

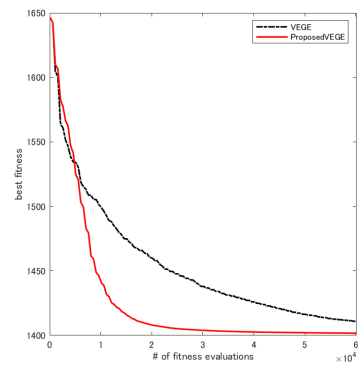

$F_{26}$

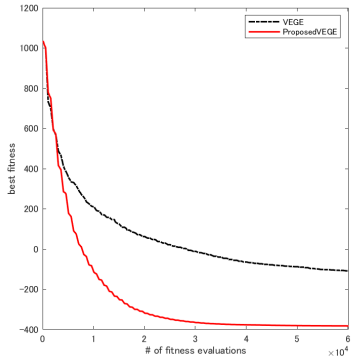

$F_{11}$

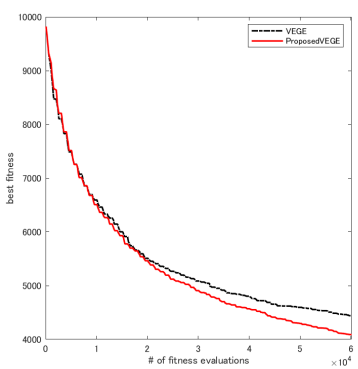

$F_{15}$

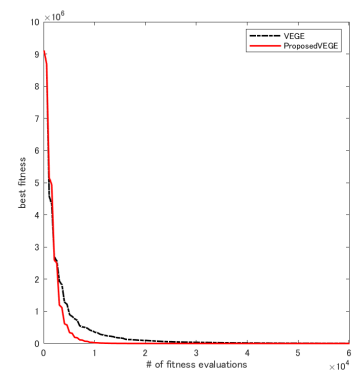

$F_{19}$

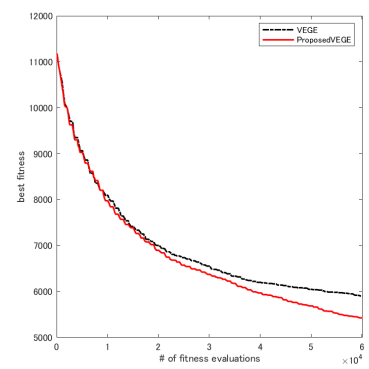

$F_{23}$

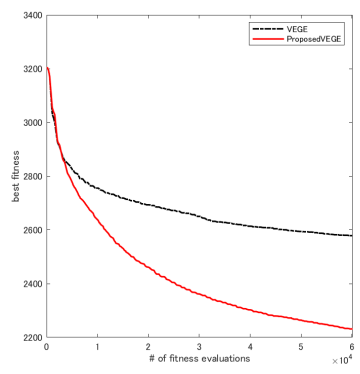

$F_{27}$

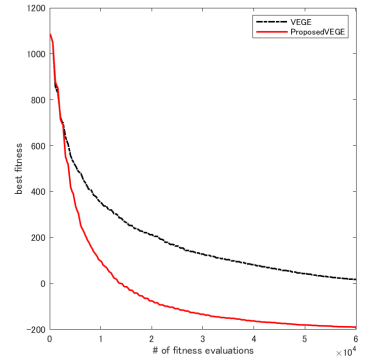

$F_{12}$

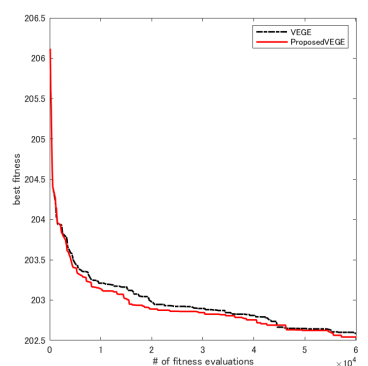

$F_{16}$

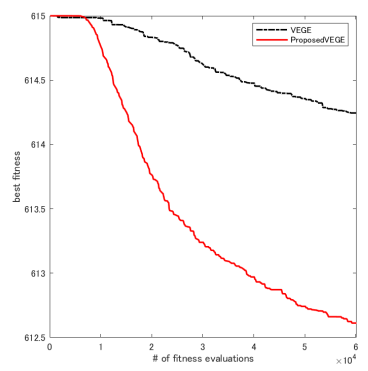

$F_{20}$

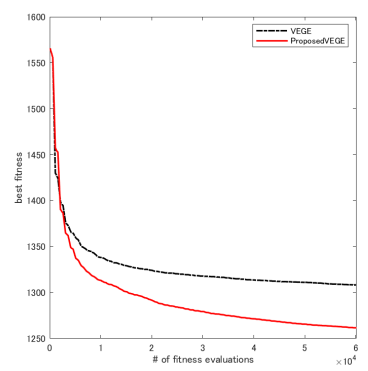

$F_{24}$

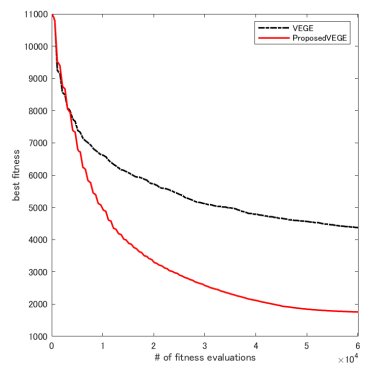

$F_{28}$

Fig. 3. Convergence curves of 30-D $F_{1}-F_{28}$ benchmark functions. We can observe that VEGE with the proposed generation strategy (red line) can accelerate VEGE search (black line). 